\title{
Tangency quantum cohomology and characteristic numbers
}

\author{
JOACHIM KOCK \\ Departamento de Matemática, Universidade Federal de Pernambuco \\ Cidade Universitária - 50670-901 Recife, PE, Brasil \\ Manuscript received on February 2, 2001; accepted for publication on April 17, 2001; \\ presented by ISRAEL VAINSENCHER
}

\begin{abstract}
This work establishes a connection between gravitational quantum cohomology and enumerative geometry of rational curves (in a projective homogeneous variety) subject to conditions of infinitesimal nature like, for example, tangency. The key concept is that of modified psi classes, which are well suited for enumerative purposes and substitute the tautological psi classes of 2D gravity. The main results are two systems of differential equations for the generating function of certain top products of such classes. One is topological recursion while the other is Witten-DijkgraafVerlinde-Verlinde. In both cases, however, the background metric is not the usual Poincare metric but a certain deformation of it, which surprisingly encodes all the combinatorics of the peculiar way modified psi classes restrict to the boundary. This machinery is applied to various enumerative problems, among which characteristic numbers in any projective homogeneous variety, characteristic numbers for curves with cusp, prescribed triple contact, or double points.
\end{abstract}

Key words: Enumerative geometry, characteristic numbers, quantum cohomology, GromovWitten invariants.

\section{INTRODUCTION}

This article exposes the main ideas and results of the theory of tangency quantum cohomology, developed in the author's PhD thesis (Universidade Federal de Pernambuco 2000), where detailed exposition, complete proofs, and a wealth of examples can be found.

\footnotetext{
*Current address: Laboratoire de Mathématiques J. A. Dieudonné, Université de Nice Sophia-Antipolis, Parc Valrose, 06108 Nice cédex 2, France

E-mail: kock@math.unice.fr
} 


\subsection{Brief overview of the SCIEnTIFIC CONTEXT}

The most classical problems of enumerative geometry are those of counting curves subject to incidence and tangency conditions; the answers to these questions are called characteristic numbers. During the last decade, the discipline has undergone a true revolution instilled by ideas from theoretical physics. The starting point was the discovery of M. Kontsevich (Kontsevich-Manin 1994) that the numbers of rational curves in $\mathbb{P}^{2}$ subject to incidence conditions are determined in all degrees by a set of differential equations which amount to the associativity of the quantum product, a new structure on the cohomology space of $\mathbb{P}^{2}$, constructed via stable maps. Kontsevich's solution to this century-old problem is unparalleled in elegance - after all, associativity is one of the most basic concepts in all of mathematics.

Since then, stable maps have been used to attack also the more difficult problem of characteristic numbers, and various solutions have been found for rational curves in $\mathbb{P}^{2}$ (or $\mathbb{P}^{r}$ ) (Pandharipande 1999, Ernström-Kennedy 1998, 1999). These solutions are quite complicated though, and the theories underlying them have little connection to physics.

The present work re-establishes that connection, making the powers of gravitational quantum cohomology available to enumerative geometry. This not only solves the rational characteristic number problem in a conceptually much simpler way, for any projective homogeneous variety, but also allows for a variety of new applications. The most surprising of the results is the construction of a tangency quantum product. This is a deformation of the usual quantum product based on modified psi classes. The associativity of this product provides a solution to the characteristic number problem which is as simple as Kontsevich's solution to the question involving only incidence conditions.

\section{MODIFIED PSI CLASSES AND DIAGONAL CLASSES}

\subsection{SET-UP}

Let $X$ be a projective homogeneous projective variety over the field of complex numbers. Fix a basis $T_{0}, \ldots, T_{r}$ for the cohomology space $H=H^{*}(X, \mathbb{Q})$.

All constructions take place in the stack $\bar{M}_{0, n}(X, \beta)$ of genus-zero $n$-pointed stable maps to $X$ of class $\beta$. Let $v_{i}: \bar{M}_{0, n}(X, \beta) \rightarrow X$ denote the $i$ 'th evaluation morphism. Pull-backs of cohomology classes of $X$ via evaluation morphisms are called evaluation classes, and top products of evaluation classes are called Gromov-Witten invariants. The reader is referred to Fulton-Pandharipande (1997) for basic properties of stable maps, Gromov-Witten invariants, and quantum cohomology.

\subsection{Psi Classes}

The tautological psi class $\psi_{i}$ is the first Chern class of the line bundle on $\bar{M}_{0, n}(X, \beta)$ whose fibre at a moduli point $[\mu: C \rightarrow X]$ is the cotangent line of $C$ at the $i$ 'th marked point. The inclusion of psi classes in the Gromov-Witten invariants corresponds in theoretical physics to introducing gravity into the quantum field theories (Witten 1991). The intersection theory of the psi classes 
goes under the name gravitational quantum cohomology (Manin 1999). The tautological psi class is not compatible with pull-back along forgetful morphisms, and for this reason it is not directly interpretable in enumerative geometry. To this end, a modification of the psi class is introduced.

\subsection{Modified Psi Classes}

The modified psi class $\bar{\psi}_{i}$ is defined for $\beta>0$ as the pull-back from the one-pointed space $\bar{M}_{0,1}(X, \beta)$ of the usual psi class $\psi_{i}$. The motivation for this definition is that many conditions from enumerative geometry allow easy expressions in terms of them, cf. $\S \S 5$ and 6 below.

\subsection{Diagonal Classes}

The $i j$ 'th diagonal class $\boldsymbol{\delta}_{i j}$ is by definition the sum of all boundary divisors having marks $p_{i}$ and $p_{j}$ together on a contracting twig. The name is justified by the following basic properties enjoyed by the diagonal classes: First, if $\pi_{i}$ is the morphism that forgets $p_{i}$ then $\pi_{i *} \delta_{i j}=1$, the fundamental class. And second,

$$
\begin{aligned}
\boldsymbol{\delta}_{i j} \boldsymbol{\delta}_{i k} & =\boldsymbol{\delta}_{i j} \boldsymbol{\delta}_{j k} \\
-\boldsymbol{\delta}_{i j}^{2}=\boldsymbol{\delta}_{i j} \overline{\boldsymbol{\psi}}_{i} & =\boldsymbol{\delta}_{i j} \overline{\boldsymbol{\psi}}_{j} .
\end{aligned}
$$

It follows that every top product involving evaluation classes, modified psi classes, and diagonal classes can be reduced to products involving only evaluation classes and modified psi classes.

\subsection{Key Formula}

The diagonal classes appear as correction terms when restricting a modified psi class to a boundary divisor $D$ both of whose twigs are of positive degree. If $D$ is the image of the gluing morphism

$$
\rho_{D}: \bar{M}_{0, n^{\prime}+1}\left(X, \beta^{\prime}\right) \times_{X} \bar{M}_{0, n^{\prime \prime}+1}\left(X, \beta^{\prime \prime}\right) \longrightarrow \bar{M}_{0, n}(X, \beta),
$$

then

$$
\rho_{D}^{*} \bar{\psi}_{i}=\overline{\boldsymbol{\psi}}_{i}+\boldsymbol{\delta}_{i \bullet}
$$

where $\bullet$ denotes the gluing mark.

\section{THE TANGENCY QUANTUM POTENTIAL AND TOPOLOGICAL RECURSION}

\subsection{The Tangency Quantum Potential}

We define an enumerative descendant to be a top product of modified psi classes and evaluation classes, and set (for $\gamma_{i} \in H$ )

$$
\left\langle\bar{\tau}_{k_{1}}\left(\gamma_{1}\right) \cdots \bar{\tau}_{k_{n}}\left(\gamma_{n}\right)\right\rangle_{\beta}:=\int \overline{\boldsymbol{\psi}}_{1}^{k_{1}} \cup v_{1}^{*}\left(\gamma_{1}\right) \cup \cdots \cup \overline{\boldsymbol{\psi}}_{n}^{k_{n}} \cup v_{n}^{*}\left(\gamma_{n}\right) \cap\left[\bar{M}_{0, n}(X, \beta)\right] .
$$


Of particular interest are the first enumerative descendants, i.e., those where the exponent of each modified psi class is at most 1 :

$$
\left\langle\overline{\boldsymbol{\tau}}_{0}^{\mathbf{a}} \overline{\boldsymbol{\tau}}_{1}^{\mathbf{b}}\right\rangle_{\beta}:=\left\langle\prod_{k=0}^{r}\left(\bar{\tau}_{0}\left(T_{k}\right)\right)^{a_{k}}\left(\bar{\tau}_{1}\left(T_{k}\right)\right)^{b_{k}}\right\rangle_{\beta} .
$$

The generating function of these invariants is called the tangency quantum potential:

$$
\Gamma(\mathbf{x}, \mathbf{y})=\sum_{\beta>0} \sum_{\mathbf{a}, \mathbf{b}} \frac{\mathbf{x}^{\mathbf{a}}}{\mathbf{a} !} \frac{\mathbf{y}^{\mathbf{b}}}{\mathbf{b} !}\left\langle\overline{\boldsymbol{\tau}}_{0}^{\mathbf{a}} \overline{\boldsymbol{\tau}}_{1}^{\mathbf{b}}\right\rangle_{\beta}
$$

Here $\mathbf{x}=\left(x_{0}, x_{1}, \ldots, x_{r}\right)$ and $\mathbf{y}=\left(y_{0}, y_{1}, \ldots, y_{r}\right)$ are formal parameters, and we employ the usual multi-index notation $\mathbf{a} !=a_{0} ! a_{1} ! \cdots a_{r} !$, and $\mathbf{x}^{\mathbf{a}}=x_{0}^{a_{0}} x_{1}^{a_{1}} \cdots x_{r}^{a_{r}}$. The variables $\mathbf{x}$ are the usual formal variables from quantum cohomology, so when $\mathbf{y}$ is set to zero, then $\Gamma$ reduces to the usual (quantum part of the) genus-zero Gromov-Witten potential.

\subsection{Deformation of the Poincaré Metric}

While the usual quantum potential is based on the trace map $\int_{\mathbf{0}}: H \rightarrow \mathbb{Q}$ (integration over the fundamental class), and the Poincaré metric $g_{i j}=\int_{0} T_{i} \cup T_{j}$, the tangency quantum potential relates more naturally to a deformation of these structures, a certain "metric" with values in $\mathbb{Q}[[\mathbf{y}]]$. The deformed trace map is

$$
\int_{\mathbf{y}} \mathbf{z}:=\sum_{\mathbf{s}} \frac{(-2 \mathbf{y})^{\mathbf{s}}}{\mathbf{s} !} \int_{\mathbf{0}} \mathbf{T}^{\mathbf{s}} \cup \mathbf{z}, \quad \mathbf{z} \in H,
$$

and the new metric is given by

$$
\gamma_{i j}:=\int_{\mathbf{y}} T_{i} \cup T_{j}
$$

Let $\left(\gamma^{i j}\right)$ denote the inverse matrix to $\left(\gamma_{i j}\right)$.

\subsection{Topological Recursion}

Since $\bar{\psi}_{i}$ admits an expression in terms of boundary divisors, a sort of topological recursion works. Note however that in contrast to the topological recursion for the usual psi classes (Witten 1991), the Key Formula 2.5 introduces a lot of diagonal classes which must then be eliminated as explained in 2.4. Miraculously, the deformed metric encodes all this combinatorics of diagonal classes. Put $\Gamma_{x_{i}}:=\frac{\partial}{\partial x_{i}} \Gamma$ and $\Gamma_{\left(x_{i} x_{j}\right)}:=\sum_{k=0}^{r} g_{i j e} g^{e f} \Gamma_{x_{f}}$ (the directional derivative with respect to $T_{i} \cup T_{j}$ ). Then, The tangency potential satisfies the differential equations

$$
\Gamma_{y_{k} x_{i} x_{j}}=\Gamma_{x_{k}\left(x_{i} x_{j}\right)}-\Gamma_{\left(x_{k} x_{i}\right) x_{j}}-\Gamma_{\left(x_{k} x_{j}\right) x_{i}}+\sum_{e, f} \Gamma_{x_{k} x_{e}} \gamma^{e f} \Gamma_{x_{f} x_{i} x_{j}} .
$$

These equations determine all first enumerative descendants from the Gromov-Witten invariants. 


\section{THE TANGENCY QUANTUM PRODUCT AND FROBENIUS STRUCTURE}

\subsection{The Tangency Quantum Product}

Define a multiplication on $H \otimes \mathbb{Q}[[\mathbf{x}, \mathbf{y}]]$ by the rule

$$
T_{i} * T_{j}:=T_{i} \cup T_{j}+\sum_{e, f} \Gamma_{i j e} \gamma^{e f} T_{f} .
$$

Here and in the sequel, for simplicity, we put $\Gamma_{i}=\Gamma_{x_{i}}$.

Now the crucial result is that this tangency quantum product is associative. The proof follows the proof of associativity of the usual quantum product (cf. Fulton-Pandharipande 1997), and again the deformed metric enters in the quadratic term to encode the combinatorics of the diagonal classes. (In the special case $X=\mathbb{P}^{2}$, this product was previously constructed by ad hoc methods in Ernström-Kennedy (1999).)

\subsection{Frobenius Structure}

From the observation $T_{i} \cup T_{j}=\sum \gamma_{i j e} \gamma^{e f} T_{f}$, it follows that if we extend the tangency quantum potential to $\beta=0$ setting

$$
\Phi:=\Gamma+\sum_{i, j, k} \frac{x_{i} x_{j} x_{k}}{3 !} \int_{\mathbf{y}} T_{i} \cup T_{j} \cup T_{k},
$$

then the tangency quantum product can be written

$$
T_{i} * T_{j}=\sum_{e, f} \Phi_{i j e} \gamma^{e f} T_{f},
$$

and the associativity equations become

$$
\sum_{e, f} \Phi_{i j e} \gamma^{e f} \Phi_{f k \ell}=\sum_{e, f} \Phi_{j k e} \gamma^{e f} \Phi_{f i \ell} .
$$

It follows readily from these constructions that The cohomology $\mathbb{Q}[[\mathbf{y}]]$-module $H[[\mathbf{y}]]$ with bilinear non-degenerate pairing $\gamma: H[[\mathbf{y}]] \otimes H[[\mathbf{y}]] \rightarrow \mathbb{Q}[[\mathbf{y}]]$, equipped with the tangency quantum potential $\Phi \in \mathbb{Q}[[\mathbf{x}, \mathbf{y}]]$ constitutes a formal Frobenius manifold over $\mathbb{Q}[[\mathbf{y}]]$. (See Manin (1999) for definitions and theory of Frobenius manifolds.)

\section{CHARACTERISTIC NUMBERS}

\subsection{TANGEnCY CONDITIONS}

Let $Z \subset X$ be a very ample hypersurface, and denote by $\eta_{i}$ the pull-back of the class of $Z$ along the $i$ 'th evaluation morphism. Now one can show that the locus of maps which are tangent to $Z$ at the $i$ 'th marked point is of class

$$
\eta_{i}\left(\eta_{i}+\bar{\psi}_{i}\right)
$$


The proof of this involves a jet-bundle construction, Porteous' formula, and a transversality argument. Furthermore, the top intersections of such loci are transverse for general hypersurfaces, so that the characteristic numbers are expressed as top products of modified psi classes and evaluation classes.

\subsection{Characteristic Number Potential and Coordinate Changes}

Let $G$ be the generating function for the characteristic numbers of rational curves in $X$. Now a combinatorial argument shows that $G$ is a linear change of coordinates of $\Gamma$. In this way, an easy application of the chain rule translates the equations of 3.3 and 4.1 into differential equations for $G$, completely determining the characteristic numbers form the numbers involving only incidence conditions (i.e., the Gromov-Witten invariants).

\subsection{Example: Plane Curves}

To give the idea, let $N_{d}(a, b, c)$ be the number of rational plane curves of degree $d$, passing through $a$ points, tangent to $b$ lines, and tangent to $c$ lines at given points (with $a+b+2 c=3 d-1$ ). The characteristic number potential

$$
G(s, u, v, w)=\sum_{d>0} \exp (d s) \sum_{a, b, c} \frac{u^{a}}{a !} \frac{v^{b}}{b !} \frac{w^{c}}{c !} N_{d}(a, b, c)
$$

is related to the tangency quantum potential $\Gamma$ by

$$
G(s, u, v, w)=\Gamma\left(x_{1}, x_{2}, y_{1}, y_{2}\right),
$$

subject to the change of variables:

$$
\begin{array}{lll}
x_{0}=0 & x_{1}=s & x_{2}=u+v \\
y_{0}=0 & y_{1}=v & y_{2}=w
\end{array}
$$

The deformed metric reads

$$
\left(\gamma^{e f}\right)=\left(\begin{array}{ccc}
0 & 0 & 1 \\
0 & 1 & 2 y_{1} \\
1 & 2 y_{1} & 2 y_{1}^{2}+2 y_{2}
\end{array}\right)=\left(\begin{array}{ccc}
0 & 0 & 1 \\
0 & 1 & 2 v \\
1 & 2 v & 2 v^{2}+2 w
\end{array}\right),
$$

and 3.3 translates into the following the differential equation satisfied by $G$.

$$
G_{v s}=G_{u s}-G_{u}+\frac{1}{2} G_{s s} G_{s s}+2 v G_{s s} G_{u s}+\left(v^{2}+w\right) G_{u s} G_{u s} .
$$

\section{FURTHER APPLICATIONS}

\subsection{Codimension-2 Corrections}

In many further applications, curves with a "marked node" contribute where they shouldn't. Let $\Pi_{1}$ denote the sum of all codimension- 2 boundary cycles whose middle twig has degree 0 and carries 
the mark $p_{1}$, while the two other twigs have positive degree. The potential for "first enumerative descendants integrated over $\Pi_{1}$ " is related to $\Gamma$ through a quadratic differential equation, since an integral over $\Pi_{1}$ can be expressed as a product of integrals over the moduli spaces corresponding to its twigs. (A similar observation applies to the potential of "first enumerative descendants integrated against a single factor $\bar{\psi}_{1}^{2}$ ": this follows from one step of topological recursion.)

\subsection{Cuspidal Curves}

Taking the above boundary classes into account, the techniques of modified psi classes also yield the characteristic numbers of cuspidal rational curves (in $\mathbb{P}^{2}$ or $\mathbb{P}^{1} \times \mathbb{P}^{1}$ ). For example, for $\mathbb{P}^{2}$, the locus of curves having cusp at the first mark is shown to be of class

$$
3 \eta_{1}^{2}+3 \eta_{1} \bar{\psi}_{1}+\bar{\psi}_{1}^{2}-\Pi_{1}
$$

In cuspidal environment, the tangency formula of 5.1 fails, because the cusp mapping to the given line will count as tangency, although it is not a limit of a honest tangency. The correct formula in this case is

$$
\eta_{i}\left(\eta_{i}+\overline{\boldsymbol{\psi}}_{i}-\delta_{1 i}\right)
$$

Now the characteristic number problem is solved like this. First, the diagonal classes are expanded using the formulae of 2.4, and next the differential equations for the enriched potentials of 6.1 are used to eliminate $\Pi_{1}$ and $\bar{\psi}_{1}^{2}$. The final result of these manipulations is three differential equations which effectively determine the cuspidal characteristic numbers from the usual ones.

\subsection{OTHER EXAMPLES}

Many other conditions allow an easy description in terms of the classes introduced above. For example, the locus of maps which have triple contact to a given hypersurface $V \subset \mathbb{P}^{r}$ is of class $\boldsymbol{\eta}_{i}\left(\boldsymbol{\eta}_{i}+\overline{\boldsymbol{\psi}}_{i}\right)\left(\boldsymbol{\eta}_{i}+2 \overline{\boldsymbol{\psi}}_{i}\right)-\left[\mathrm{I}_{i}\right]_{3}-\boldsymbol{\eta}_{i} \Pi_{i}$. (Here $\left[\mathrm{I}_{i}\right]_{3}$ denotes a certain correction consisting of two-twig maps with a linear twig mapping into $V$, but it turns out never to give any contribution.) The locus of maps which are secant to a codimension-2 plane (at $p_{1}$ and $\left.p_{2}\right)$ is of class $\boldsymbol{\eta}_{1}^{2} \boldsymbol{\eta}_{2}^{2}-\boldsymbol{\delta}_{12}\left(2 \boldsymbol{\eta}_{1}^{3}+\right.$ $\left.\eta_{1}^{2} \bar{\psi}_{1}\right)-\eta_{1}^{2} \Pi_{12}$. (For the plane, this is the locus of curves with specified double point.) Cutting this locus with $\delta_{12}$ forces the two secant points together, and we find the class of being tangent to the codimension-2 plane.

\section{ACKNOWLEDGEMENTS}

The author's PhD studies were financed by the Natural Science Research Council of Denmark, which is gratefully acknowledged. The author is also thankful to the Departamento de Matemática da Universidade Federal de Pernambuco for four lovely years, and in particular to his thesis advisor Israel Vainsencher for precious guidance and encouragement. 


\section{RESUMO}

Este trabalho estabelece uma conexão entre co-homologia quântica gravitacional e geometria enumerativa de curvas racionais (em uma variedade homogênea projetiva) sujeita a condições de natureza infinitesimal como, por exemplo, tangência. O conceito chave é de classes psi modificadas, que são bem apropriadas para propósitos enumerativos e substitui as classes psi tautológicas de gravidade 2D. Os resultados principais são dois sistemas de equações diferenciais para a função geradora de certos produtos de ordem superior de tais classes. Um é recorrência topológica enquanto o outro é Witten-Dijkgraaf-Verlinde-Verlinde (WDVV). Em ambos os casos, entretanto, a métrica Riemanniana não é a métrica usual de Poincaré, mas, uma certa deformação desta que, surpreendentemente, codifica todas as possibilidades combinatórias do modo peculiar pelo qual classes psi modificadas restringem-se ao bordo. Esta maquinaria é aplicada a vários problemas enumerativos, entre os quais números característicos em qualquer variedade homogênea projetiva, números característicos para curvas com cúspides, contato triplo prescrito, ou pontos duplos.

Palavras-chave: geometria enumerativa, números característicos, co-homologia quântica, invariantes Gromov-Witten.

\section{REFERENCES}

ERNSTRÖM L AND KENNEDY G. 1998. Recursive formulas for the characteristic numbers of rational plane curves. J Alg Geom 7: 141-181. (alg-geom/9604019).

ERnström L and Kennedy G. 1999. Contact cohomology of the projective plane. Amer J Math 121: 73-96. (alg-geom/9703013).

Fulton W and Pandharipande R. 1997. Notes on Stable Maps and Quantum Cohomology. In J. Kollár, R. LAZARsfeld and D. Morrison, editors, Algebraic Geometry, Santa Cruz 1995, vol. 62, II of Proc Symp Pure Math, pp. 45-96. (alg-geom/9608011).

Kоск J. 2000. Tangency quantum cohomology and enumerative geometry of rational curves. PhD thesis, Recife, Brazil, March. Available at http://www-math.unice.fr/ kock/GW/TESE.ps.

Kontsevich M And Manin YuI. 1994. Gromov-Witten classes, quantum cohomology, and enumerative geometry. Comm Math Phys 164: 525-562. (hep-th/9402147).

MANIN YuI. 1999. Frobenius manifolds, quantum cohomology, and moduli spaces. AMS Colloquium Publications, Providence, RI.

PANDHARIPANDE R. 1999. Intersections of $\mathbb{Q}$-divisors on Kontsevich's moduli space $\bar{M}_{0, n}\left(\mathbb{P}^{r}, d\right)$ and enumerative geometry. Trans Amer Math Soc 351: 1481-1505. (alg-geom/9504004).

WitTEn E. 1991. Two-dimensional gravity and intersection theory on moduli space. Surveys in Diff Geom 1: 243-310. 